九州大学学術情報リポジトリ

Kyushu University Institutional Repository

\title{
Über Die Oxydation Von Xylan Mit Perjodat
}

Hamada, Matsukichiro

Laboratory of Pesticide Chemistry, Faculty of Agriculture, Kyushu University

Maekawa, Kazuyuki

Laboratory of Pesticide Chemistry, Faculty of Agriculture, Kyushu University

https://doi.org/10.5109/22635

出版情報: 九州大学大学院農学研究院紀要. 9 (4), pp.311-316, 1950-11. Kyushu University バージョン：

権利関係 : 
journai of the Facuity of Agricuiture, Kyūshū Unniversity, Vol. $\mathcal{Y}_{2}$ N No. 4 November 30,1950

\section{ÜBER DIE OXYDATION VON XYLAN MIT PERJODAT*}

\section{Matsukichiro Hamada und Kazliyuki Maekawa}

Über die Verwertung von Xylose und seinem Polymere durch Tiere bzw. Mikroben sind schon einige Ergebnisse berichtet worden ${ }^{1,2,3}$. Die Konfiguration von Xylan war durch Haworth und seine Mitarbeiter $4,5,6,7>$ wie folgend festgestellt worden, dass Xylopyranosceinheiten sich an ihrer 1,4 Stelle und in 3-Typus verbinden. Den Polymerisationsgrad der Xyloseanhydridreste im Xylan hat Husemann ${ }^{3}$ als beinahe 150 berichtet.

Wenn Xylan die Perjodat-Oxydation, sog. Glykol-Spaltung erleiden kann, so wird bis auf weiteres vermutet, dass diese

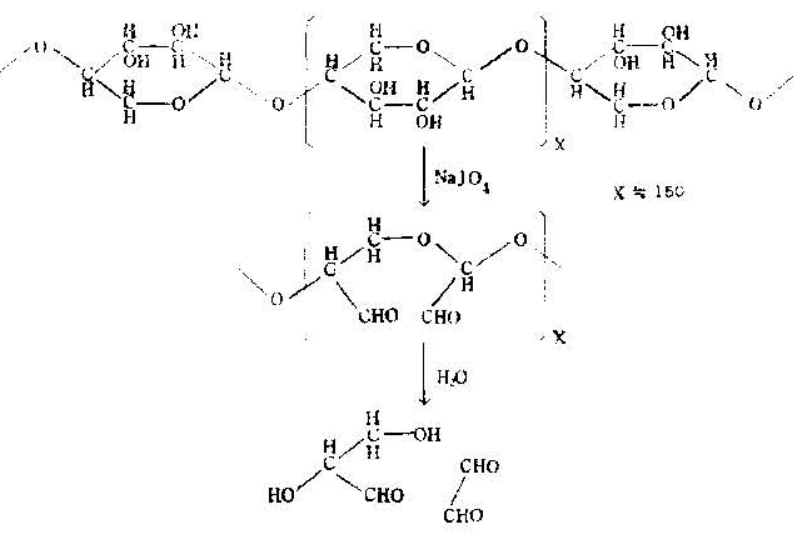

Spaltung zwischen der einzigen benachbarten Hyrlroxyl-Gruppe habenden Kohlenstoffatomen der Xyloseanhydridreste, C 2-C 3,

* Vorgetragen vor dem Bezirksverein West-Japan von der Agr. Chem. Soc. Japan atm 7. Juni 1947. Refr., J. Iap. Soc. Food \& Nutrition, 1 (1947), 28.

$\dagger$ Nachherige Anmerkung; Die Arbeiten von G. Jayme und M. Särtre "Über die Oxydation des Xylans mit Perjodsature. Ber., 75 (1942), 1840; ibid.. 77 (1944), 242; $248^{\prime \prime}$ sini utus urst nach Fertigstellung dieser Mitteilung 13. Sept. 19.17; bekannt geworden. 
stattfinden kann, und dass Glycerinaldehyd und Glyoxal bei der Hydrolyse des mit Perjodate aus Xylan erhaltenen Oxyxylans entstehen kann.

Wenn man nach diesem Verfahren aus Xylan Glycerinaldehyd gewinnen kann, obgleich je zwei Kohlenstoffatome aus $\mathrm{C}_{5}$-Einheit von den Xyloseanhydridresten verloren gehen, so ist dies sowohl eine neue Erkenntnis, als auch ein gewisser Beitrag zur Lösung des Ernährungsproblems.

Wir beschäftigten uns also mit Perjodat-Oxydation von Xylan und, wie erwartet, konnten wir aus dem Hydrolysate des Oxyxylans Glycerinaldehyd mit guter Ausbeute isolieren.

\section{BESCHREIBUNG DER VERSUCHE}

I. Spaltung des Xylans mit Natrium-meta-Perjodat.

Xylan, das aus Weizen- und Reisstroh nach gewöhnlicher Weise ${ }^{\pi, 6}$ gewonnen worden war, war in seinem Pentosangehalte $92,6 \%$. $6.6 \mathrm{~g}\left(1 / 20\right.$ von $\mathrm{C}_{s}$-Einheitsgewicht $)$ dieser Probe wurde in

Fig. 1

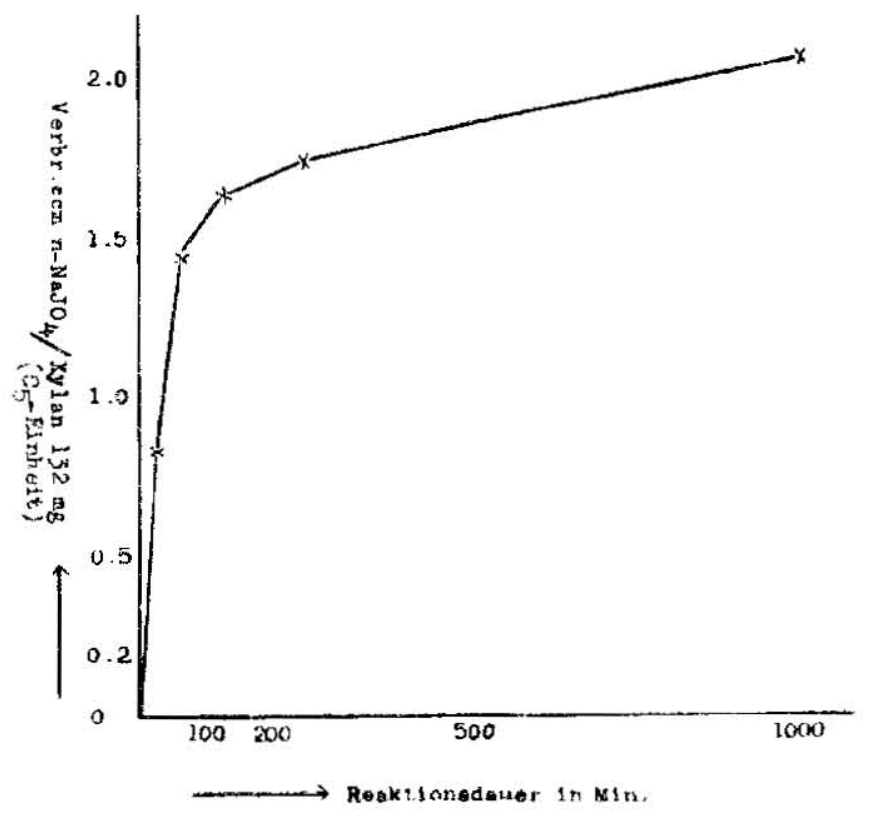


$500 \mathrm{ccm}$ Glasstöpselflaschen abgewogen, hierzu wurden $300 \mathrm{ccm}$ $0.383 \mathrm{n}$-Natriumperjodat (1.15 Äquivalenz für $\mathrm{C}_{5}$-Einheit von Xylan? zugesetzt, dieses wurde bei Zimmertemperatur unter gelegentlichem Erschüttern belassen, bis sich die erforderliche Reaktion vollzog. Die Oxydation ging während der ersten zwei Stunden verhältnismässig schnell vor sich, es bedurfte aber 15 Stunden, un 2-Äguivalenz von Perjodate zu verbrauchen (Fig. 1).

Es wurde dann durch Kollodiummembran dialysiert, um anorganische Substanzen zu entfernen, und in Vakuum bis $50 \mathrm{ccm}$ eingedampft. Zwei Volumen Aceton zur letzteren hinzugegossen, bewirkten weissen Niederschlag, der aus Oxyxylan bestand. Ausbeute $4.6 \mathrm{~g}$.

II. Eigenschaften des oxydierten Xylans.

Diese oxydierte Substanz war dem anfänglichem Xylan anscheinend gleich, aber in gewisser Weise spröde, überdies ergab dies nach Tollens'schem Verfahren für Pentosan-Bestimmung fast kein Furfural, vielmehr reduzierte Fehling'sche Lösung und ziemlich viel Jod, konderssierte mit Phenol und Anilin und zeigte grösser Alkalizahl ${ }^{15}$. Diese Tatsache wurde in Tafel 1 zusammengefasst.

Tafel 1.

\begin{tabular}{l|ll}
\hline & Xylan & Oxyxylan \\
\hline $\begin{array}{c}\text { Pentosangehalt als Phloroglucid } \\
\text { (Tollens'sches Verf.) }\end{array}$ & $92.6 \%$ & spur \\
$\begin{array}{l}\text { Reagiertes Phenol (Pro } 132 \mathrm{mg}) \\
\text { Reagiertes Anilin (Pro } 132 \mathrm{mg})\end{array}$ & $64.4 \mathrm{mg}$ & $.361 .8 \mathrm{mg}$ \\
$\begin{array}{l}\text { Iodverbr. nach Willstäter und Schudel. } \\
\text { schem Verf. (ccm 0.1 } \mathrm{n} \text { Jod/132 mg) }\end{array}$ & spur & 30.01 \\
Alkalizahl nach Schoch u. Jensen & 28 & 54 \\
\hline
\end{tabular}

Aus der Differenz der Daten für Kontrollansatz und Hauptversuch ist leicht zu erkennen, dass die Oxy-Probe eigentümlicherweise reich an Aldehyd ist. 
III. Hydrolysc: von oxydiertem Xylan.

4 g oben erwähntes Oxyxylan wurde in gut schliessenden Glasstöpselflaschen in $150 \mathrm{ccm}$ Wasser suspendiert und mit $50 \mathrm{ccm}$ $1.5 \mathrm{n}$-Schwefelsäure versetzt. Die Hydrolyse wurde in kochendem Wasserbad nach 30-stündigem Aufbewahren vollzogen. Das dabei entstandenc wenige Harz und Unlösliches wurden entfernt und betrugen etwa $0.2 \mathrm{~g}$.

\section{(i) Glyoxal aus dem Hydrolysate}

(a). $10 \mathrm{ccm}$ obiges Hydrolysat wurde mit einer Lösung von $0.4 \mathrm{~g} \mathrm{2,4-Dinitrophenylhydrazin} \mathrm{in} 10 \mathrm{ccm}$ Eisessig versetzt. Nach einstündigem Erhitzen in der geschlossenen Röhre im Wasserbad wurde der entstandene gelb-orange Niederschlag abgesaugt, erst mit schwacher Fissigsäure, dann mit Wasser gewaschen und im Vakuumexsikkator getrocknet. Die Ausbeute betrug $0.408 \mathrm{~g}=$ $63.4 \%$ der Theorie. Nach Umkristallisieren aus Essigsäureanhydrid zeigte die Substanz den unveränderlichen Schmelzpunkt $322^{\circ}$ unter Zersetzen $^{10,11)}$.

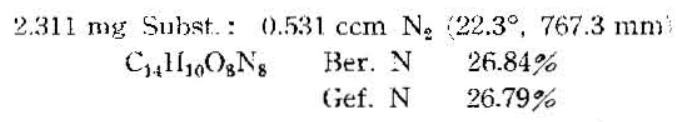

(b). Von einem anderen der obigen Hydrolysate entfernte man die Schwefelsäure mit Bariumcarbonat, $20 \mathrm{ccm}$ dieses neutralen Hydrolysates wurden mit einer Lösung von $0.7 \mathrm{~g}$ Phenylhydrazin in $10 \mathrm{ccm}$ Äthanol vermischt. Es entstand sofort ein gelber Niederschlag, der bei Erhitzen bald orange wurde, das Gemisch wurde nunmehr eine Stunde lang erhitzt. Die Kristalle wurden abgesaugt und mit Wasser gewaschen, sowie im Vakuum getrocknet. Frhalten wurden $0.4492 \mathrm{~g}$. Osazon $=43.2 \%$ der Theorie, nach zweimaligem Umkristallisieren aus Äthanol zeigte die Substanz den Schmp. $169^{\circ 12,13,14,15)}$. Die Verbindung bildet gelbe warzenförmige Aggregate.

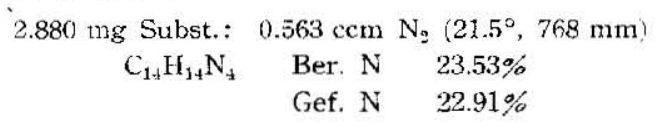

Beide wurden durch Mischprobe als Glyoxalderivate testgestellt.

(ii) Glycerinaldehyd aus dem Hydrolysate 
Das rückbleibende neutrale Hydrolysat wurde nach Entfärbung mittels Tierkohle zuerst im Vakuum bei $35^{\circ}$, dann über Schwefelsäure im Exsikkator verdampft. Der völlig farblose Sirup erstarrte nach 4 Wochen $z$ e einem Brei weisser Kristalle. Nach Umkristallisieren aus 50\% igem Methanol zeigte es den Schmp. 136 $6^{\left.{ }^{16}\right)}$. Ausbeute $2 \mathrm{~g}$. Dies bildet farblose Nadeln, die wie die Kohlenhydrate im allgemeinen in organischen Solventien schwer, in Wasser ziemlich löslich sind. Diese Substanz zeigte PhloroglucinReaktion von Glycerinaldehyde nach Wohl und Neuberg ${ }^{16)}$ bzw. Resorcin, Thymol, $\beta$-Naphtol-Reaktion nach Denigès ${ }^{17}$ ) jede für sich stark.

(a) Benzoylierung des Glycerinaldehyds; $0.5 \mathrm{~g}$ fein gepulverter Glycerinaldehyd (oben erwähnte) wurden in $30 \mathrm{ccm}$ Pyridin suspendiert und mit $3.5 \mathrm{~g}$ Benzoylchlorid versetzt, wobei erhebliche Erwärmung eintrat. Am nächsten Tage wurde die Hauptmenge des Pyridins im Vakuum abdestilliert, der dünne Sirup mit Eiswasser durchgeknetet und einige Stunden unter wiederholter Erneuerung des Wassers der Kristallisation überlassen. Ausbeute $1.8 \mathrm{~g}$. Das Benzoat ist etwas leichter löslich in Äthanol, nach 4maligem Umkristallisieren aus Äthanol zeigte es den Schmp. $80^{\circ 18}$.

(b) 2,4-Dinitro-phenylhydrazon des O-Benzoyl-Glycerinaldehyds; $0.3 \mathrm{~g}$ benzoylierten Glycerinaldehyd wurden mit $0.5 \mathrm{~g} \mathrm{2,4-}$ Dinitro-phenylhydrazin in $30 \mathrm{ccm}$ Äthanol und $3 \mathrm{ccm}$ Eisessig vermischt. Nach einiger Zeit begann die Kristallisation des gelben Hydrazons, während die roten Nadeln des Hydrazins allmählich verschwanden. Zur Vervollständigung der Umsetzung wurde es nach etwa 30 Min. kurz aufgekocht. Rohprodukt $0.4 \mathrm{~g}$. Nach Reinigung aus Eisessig zeigte es den Schmp. 177' (unkorr.).

$$
\begin{array}{ccc}
3.256 \mathrm{mg} \text { Subst.: } & 0.255 \mathrm{ccm} \mathrm{N} & \left(22.3^{\circ}, 767.3 \mathrm{~mm}\right) \\
\mathrm{C}_{\mathrm{n}_{3} \mathrm{H}_{18} \mathrm{O}_{8} \mathrm{~N}_{4}} & \text { Ber. N } & 9.18 \% \\
& \text { Gef. N } & 9.13 \%
\end{array}
$$

Betreffs Oxyxylans und seines Hydrolysierens geht es um folgende Ziffern.

Tafel 2.

\begin{tabular}{c|c|c|c|c}
\hline Xylan & Oxyxylan & Glycerinaldehyd & $\begin{array}{c}\text { Glyoxal als } \\
\text { 2, 4-Dinitro- } \\
\text { phenylosazon }\end{array}$ & Phenylosazon \\
\hline $\begin{array}{c}6.6 \mathrm{~g} \\
92.6 \%)\end{array}$ & $4.6 \mathrm{~g}$ & $2.0 \mathrm{~g}$ & $63 \%$ & $43 \%$ \\
\hline
\end{tabular}


Dieser Versuch wurde mit Hilfe der Ausgaben des Unterrichtsministeriums für Wissenschaftliche Forschung durchgeführt, wofür die Verfasser an dieser Stelle Dank sagen möchten.

\section{LITERATUR}

(1) M. M. Miller u. H. B. Lewis; J. biolog. Chem., 98 (1932), 133; 141.

(2) H. Iwata; Bull. Imp. Coll. Agric. Forest., (Morioka), 21 (1935), 1.

(3) W. Voss u. G. Butter; Ann., 534 (1938), 161.

(4) W. Voss, R. Bauer u. J. Pfirschke; Ann., 534 (1338), 135.

(5) H. A. Hampton, W. N. Haworth u. E. L. Hirst; Soc., 1929, 1739.

(6) R. A. S. Bywater, W. N. Haworth u. S. Peat; Soc., 1937, 1983.

(7) W. N. Haworth u. E. G. Percival; Soc., 1931, 2850.

(8) E. Iusemann; Naturw., 27 (1939), 595; J. prakt. Chem., 155 (1940), 13.

(9) T. J. Schoch u. C. C. Jensen; Ind. Eng. Chem. Anal, Ed., $12(1940), 531$.

(10) S. Gladstone u. A. Hickling; Soc., 1936, 824.

(11) H. J. Lucas, A. N. Prater u. R. E. Morris ; Am., 57 (1935), 725.

(12) C. S. Hudson u. Mitarb; Am., 59 (1937), 2049.

(13) C. S. Hudson u. Mitarb; ibid., 60 (1938), 989.

(14) K. Hess u. Cl. Uibrig; Ber., 50 (1917), 367.

(15) E. Fischer; Ber., 17 (1884), 5;5.

(16) A. Wohl u. C. Neuberg; Ber., 33 (1900), 3095.

(17) G. Denigès; Bull., (4) 5 (1909), 878; C. A. 3 (1909), 1163.

(18) P. Brigl u. H. Grüner; Ber., 66 (1933), 931. 